\title{
Prostaglandin D Synthase ( $\beta$-Trace) in Human Arachnoid and Meningioma Cells: Roles as a Cell Marker or in Cerebrospinal Fluid Absorption, Tumorigenesis, and Calcification Process
}

\author{
Tetsumori Yamashima, ${ }^{1}$ Kazushige Sakuda, ${ }^{1}$ Yasuo Tohma, ${ }^{1}$ Junkoh Yamashita, ${ }^{1}$ Hiroshi Oda, ${ }^{2}$ \\ Daisuke Irikura, ${ }^{3}$ Naomi Eguchi, ${ }^{4}$ Carsten T. Beuckmann, ${ }^{3}$ Yoshihide Kanaoka, ${ }^{3}$ Yoshihiro Urade, ${ }^{3}$ \\ and Osamu Hayaishi ${ }^{3}$ \\ ${ }^{1}$ Department of Neurosurgery, Kanazawa University School of Medicine, Kanazawa 920, Japan, 2Department of \\ Biological Chemistry, Central Research Institute, Maruha Corporation, Tsukuba 300-42, Japan, ${ }^{3}$ Department of Molecular \\ Behavioral Biology, Osaka Bioscience Institute, Osaka 565, Japan, and ${ }^{4}$ Department of Precursory Research for \\ Embryonic Science and Technology, Osaka 590-02, Japan
}

\begin{abstract}
Glutathione-independent prostaglandin D synthase (PGDS) is an enzyme responsible for biosynthesis of prostaglandin $D_{2}$ in the CNS and is identical to a major cerebrospinal fluid protein, $\beta$-trace. Although PGDS has been identified recently in rat leptomeninges, little information is available about human meninges or meningiomas. Here, we report PGDS to be expressed consistently in 10 human arachnoid and arachnoid villi and in 21 meningiomas by immunohistochemistry, Western blot, and reverse transcription (RT)-PCR analyses. In arachnoid, PGDS immunoreactivity was seen in arachnoid barrier cells but was negligible in arachnoid trabecula and pia mater. In contrast, in arachnoid villi, PGDS was seen in core arachnoid cells rather than in the cap cell cluster or arachnoid cell layer. Meningioma cells also showed intense immunoreactivity in the perinuclear region, and it was often concentrated within meningocytic whorls and around calcifying psammoma bodies. Immunoelec-
\end{abstract}

Since the discovery of prostaglandin (PG) in 1930, various types of PGs have been demonstrated throughout almost all types of cells in mammalian organs and tissues. PGs are well known to play vital roles in contraction and relaxation of smooth muscles, aggregation and disaggregation of platelets, inflammation, and pain.

$\mathrm{PGD}_{2}$ had long been considered a minor and biologically inactive prostanoid. In the late 1970s, Hayaishi and his co-workers found a large amount of $\mathrm{PGD}_{2}$ in the brains of rats and other mammals, including humans (for review, see Hayaishi, 1994). In rats (Ueno et al., 1983), monkeys (Onoe et al., 1988), and humans (Hayaishi, 1988), $\mathrm{PGD}_{2}$ may be an endogenous sleep-promoting factor, which circulates in the ventricular system, subarachnoid space, and extracellular space of the brain and interacts with receptors on the ventromedial surface of the rostral basal forebrain to initiate the signal to let the brain sleep (Matsumura et al., 1994).

Glutathione-independent PGD synthase (PGDS) [(5Z,13E)(15S)-9 $\alpha, 11 \alpha$-epidioxy-15-hydroxyprosta-5,13-dienoate D-isomerase, EC 5.3.99.2] is an enzyme that catalyzes the conver-

Received Sept. 26, 1996; revised Dec. 18, 1996; accepted Jan. 9, 1997.

Correspondence should be addressed to Tetsumori Yamashima, Department of Neurosurgery, Kanazawa University School of Medicine, Takaramachi 13-1, Kanazawa 920, Japan.

Copyright $\Subset 1997$ Society for Neuroscience $0270-6474 / 97 / 172376-07 \$ 05.00 / 0$ tron microscopic data, when compared with the ultrastructure, showed that PGDS was localized at rough endoplasmatic reticulum of arachnoid and meningioma cells. Western blot showed a $29 \mathrm{kDa}$ immunoreactive band indicating PGDS, but the extent of expression was variable from case to case, which was compatible with immunohistochemical data. RT-PCR revealed PGDS gene expression in all meningiomas studied, regardless of histological subtypes, and also in human arachnoid villi. Because human arachnoid and meningioma cells exclusively express PGDS, it can be considered their specific cell marker. These results show functional differences in various types of meningeal cells attributable to differences in PGDS expression.

Key words: arachnoid; arachnoid villus; meningioma; prostaglandin D synthase; $\beta$-trace; prostaglandin; cerebrospinal fluid

sion of $\mathrm{PGH}_{2}$ to $\mathrm{PGD}_{2}$ in the presence of various sulfhydryl compounds (Urade et al., 1985). This enzyme belongs to the lipocalin superfamily (Nagata et al., 1991; Peitsch and Boguski, 1991) and shares characteristics similar to those of other lipocalins. It is secreted into the cerebrospinal fluid (CSF), where it is identical to $\beta$-trace (Clausen, 1961; Kuruvilla et al., 1991; Hoffmann et al., 1993; Zahn et al., 1993; Watanabe et al., 1994), and binds retinoids or other lipophilic substances with affinities similar to those of other lipocalins (Urade, 1996). Accordingly, $\beta$-trace or PGDS is a major CSF protein.

We have demonstrated both this enzyme and its mRNA in the leptomeninges, choroid plexus, and retina of rats (Urade et al., 1993; Beuckmann et al., 1996). Accordingly, it is probable that human arachnoid cells might synthesize and secrete this enzyme into the subarachnoid space. In this paper, we have studied human arachnoid, arachnoid villi, and meningiomas by means of immunohistochemistry, immunoelectron microscopy, Western blot, and reverse transcription (RT)-PCR. Human arachnoid and meningioma cells exclusively expressed PGDS, which can be used as their specific cell marker. Furthermore, we detected differences in PGDS expression among different types of arachnoid cells, which until now have been characterized only morphologically. The possible roles of PGDS or $\mathrm{PGD}_{2}$ in CSF absorption, meningioma biology, or calcification process are discussed. 


\section{MATERIALS AND METHODS}

Human materials. Both arachnoid and arachnoid villi were obtained from 10 cases (eight male and two female with an average age of 47 years) at autopsy within a few hours postmortem or at surgery and were used for immunohistochemical and immunoelectron microscopic analyses. A fresh sample of arachnoid villi obtained at surgery was used for RT-PCR analysis. The samples of 19 meningiomas histologically comprising five syncytial, five transitional, one fibroblastic, one angiomatous, and seven atypical types were obtained fresh at surgery and were used for immunohistochemical, immunoelectron microscopic, Western blot, and RTPCR analyses. Furthermore, two typical psammomatous or ossifying meningiomas, including numerous psammoma bodies or ossification deposits, were used for immunohistochemical analysis.

For immunohistochemistry, the samples were fixed with $3.7 \%$ formalin or $4 \%$ paraformaldehyde and embedded in paraffin. For immunoelectron microscopy, the samples were fixed with $4 \%$ paraformaldehyde and processed for pre-embedding procedure. The samples of meningiomas obtained at surgery for Western blot and RT-PCR analyses were frozen and stored.

Antiserum and primers. According to the methods described previously for rabbit anti-rat PGDS serum (Urade et al., 1985), polyclonal antibody against PGDS of the human brain type was elicited in rabbits for immunohistochemistry and Western blot. Briefly, PGDS was purified from human CSF to apparent homogeneity (Watanabe et al., 1994), and the purified enzyme $(500 \mu \mathrm{g})$ was emulsified with TiterMax adjuvant (Vaxcel, Norcross, GA) and injected subcutaneously into a rabbit weighing $\sim 2 \mathrm{~kg}$. The booster injections $(250 \mu \mathrm{g}$ of enzyme) were carried out four times at 3 week intervals. The rabbit was bled through the carotid artery $7 \mathrm{~d}$ after the last injection to recover the antiserum.

The oligonucleotide primers used for PCR amplification were as follows: PGDS (5'-primer, 5'-GACAAGTTCCTGGGGCGCTGGT-3'; 3' primer, 5'-GCTGTAGAGGGTGGCCATGC-3') and glyceraldehyde 3-phosphate dehydrogenase (GAPDH) (5'-primer, 5'-CATGACCACAGTCCATGCCATCACT-3'; 3'-primer, 5'-TGAGGTCCACCACCCTGTTGCTGTA-3').

Immunohistochemistry. Sections of $4 \mu \mathrm{m}$ thickness were incubated overnight at $4^{\circ} \mathrm{C}$ in anti-PGDS serum diluted 1:5000. The immunoreaction was visualized by the immunoperoxidase method using anti-rabbit IgG and avidin-biotin-peroxidase complex (Vector, Burlingame, CA). Then, the sections were immersed in a solution of $500 \mu \mathrm{g} / \mathrm{ml}$ diaminobenzidine hydrochloride (DAB) (Sigma, St. Louis, MO) in $50 \mathrm{~mm}$ Trisbuffered saline (TBS, pH 7.6) for $30 \mathrm{sec}$. The controls for immunohistochemistry were made using primary antibody preabsorbed with a threefold volume of recombinant $\beta$-trace and nonimmunized rabbit serum.

Immunoelectron microscopy. Vibratome sections of $50 \mu \mathrm{m}$ thickness were incubated for $15 \mathrm{~min}$ with $0.02 \%$ saponin for etching. Washings between each step were performed with $50 \mathrm{~mm}$ TBS. The sections were incubated with $0.3 \% \mathrm{H}_{2} \mathrm{O}_{2}$ in methanol for $1 \mathrm{hr}$ and with blocking antibody for $2 \mathrm{hr}$. The sections were then incubated with anti-PGDS serum diluted 1:3000 overnight at $4^{\circ} \mathrm{C}$. The sections were subsequently incubated with biotinylated antibody against rabbit $\mathrm{IgG}$ for $2 \mathrm{hr}$ and were incubated with avidin-biotin-peroxidase complex for $2 \mathrm{hr}$. The sections were fixed with $0.1 \%$ glutaraldehyde for $30 \mathrm{~min}$ and immersed in a solution of $500 \mu \mathrm{g} / \mathrm{ml} \mathrm{DAB}$ in TBS for $30 \mathrm{~min}$ and further in the same solution containing $0.03 \% \mathrm{H}_{2} \mathrm{O}_{2}$ for $15 \mathrm{~min}$. The sections were post-fixed with $1 \% \mathrm{OsO}_{4}$ for $60 \mathrm{~min}$, dehydrated by passage through an ascending series of ethanol, and embedded in an Epon-Araldite mixture. Ultrathin sections were cut on an LKB ultramicrotome and examined with Hitachi H-600 electron microscope without counterstaining.

The controls for immunoelectron microscopy were made using nonimmunized rabbit serum as primary antibody.

Electron microscopy. Biopsy specimens of meningiomas were fixed in cacodylate-buffered $2.5 \%$ glutaraldehyde for $2 \mathrm{hr}$ and post-fixed in $1 \%$ $\mathrm{OsO}_{4}$ in the same buffer for $1 \mathrm{hr}$. They were subsequently dehydrated through graded concentrations of ethanol and were embedded in an Epon-Araldite mixture. Semithin sections were cut with an LKB ultramicrotome and stained with toluidine blue. Ultrathin sections of selected areas were stained with uranyl acetate followed by lead citrate and were examined with a Hitachi H-600 electron microscope.

Western blot. After a protein assay, samples of meningiomas were subjected to SDS-PAGE using a ready-made polyacrylamide gel Multi Gel 15/25 (Daiichi Pure Chemicals, Tokyo, Japan). Then, proteins were transferred to polyvinylidene difluoride membranes (Atto, Tokyo, Japan) for immunoblotting. The primary anti-PGDS antibody was diluted
1:5000. The secondary antibody, peroxidase-conjugated goat anti-rabbit IgG (Sigma), was diluted 1:10,000 in $20 \mathrm{~mm}$ Tris/ $\mathrm{HCl}$ buffer containing $1 \%$ bovine serum albumin and $0.1 \%$ Tween 20 . Immunoreactive species were visualized using an alkaline phosphatase substrate kit (Vector).

$R T-P C R$. Total cytoplasmic RNA was isolated by the acid guanidine thiocyanate-phenol-chloroform method (Chomczynski and Sacchi, 1987) using Isogen (Nippon Gene, Toyama, Japan). RT-PCR was performed using an RNA PCR Kit Version 2 (Takara Shuzo, Ohtsu, Japan) according to the manufacturer's protocol.

For RT, total RNA $(1 \mu \mathrm{g})$ was mixed in a $20 \mu$ l reaction mixture containing $1 \mathrm{~mm} \mathrm{MgCl} 2,400 \mu \mathrm{M}$ dNTP mixture, $1 \mathrm{U}$ RNase inhibitor, 125 $\mathrm{nM}$ oligo(dT) $)_{20} \mathrm{M} 4$ adaptor primer, and $0.25 \mathrm{U}$ avian myeloblastosis virus reverse transcriptase XL (Life Sciences, Hialeah, FL) in RNA PCR buffer $(10 \mathrm{mM}$ Tris/ $\mathrm{HCl}$ and $50 \mathrm{mM} \mathrm{KCl})$. After synthesis of first-strand cDNA at $42^{\circ} \mathrm{C}$ for $15 \mathrm{~min}$, a denaturation step at $99^{\circ} \mathrm{C}$ for $5 \mathrm{~min}$ was performed. For PCR, the template $(2 \mu \mathrm{l})$ was subjected to amplification in a $50 \mu \mathrm{l}$ PCR reaction mixture containing $40 \mu \mathrm{M}$ dNTP mixture, $2 \mathrm{pM}$ of each PCR-primer for PGDS or GAPDH, and 0.1 U Taq polymerase (Takara Shuzo) in RNA PCR buffer. Denaturation was carried out at $94^{\circ} \mathrm{C}$ for 105 sec, and amplification was carried out for 25 cycles at $94^{\circ} \mathrm{C}$ for $30 \mathrm{sec}$ and at $68^{\circ} \mathrm{C}$ for $90 \mathrm{sec}$ in an automated DNA Thermal Cycler (GeneAmp PCR System 2400, Perkin-Elmer, Norwalk, CT). After amplification, the RT-PCR reaction mixture $(10 \mu \mathrm{l})$ was electrophoresed on a $2 \%$ agarose gel which was visualized by ethidium bromide staining with an ultraviolet transilluminator (FAS II, Toyobo, Osaka, Japan).

\section{RESULTS}

\section{Light and electron microscopic localization of PGDS Arachnoid}

PGDS was immunohistochemically demonstrated in all of the 10 arachnoid samples studied. PGDS immunoreactivity was intense in arachnoid barrier layer and weak in the underlying arachnoid cells, but was negligible in dural border cells, arachnoid trabecula, pia mater, and the underlying brain. PGDS immunoreactivity was seen as granular DAB reaction products in the cytoplasm of arachnoid barrier cells (Fig. 1A,C).

By immunoelectron microscopy, PGDS was demonstrated to be localized at the rough endoplasmatic reticulum (ER) membrane (Fig. 1D), when it was compared with the conventional ultrastructural feature (Fig. $1 F$ ). Aggregates of DAB reaction products were often seen within the cisternae of rough ER and appeared to be consistent with granular $\mathrm{DAB}$ reaction products observed by light microscopy.

Arachnoid barrier cells were ultrastructurally abundant with enlarged rough ER (Fig. 1F).

\section{Arachnoid villi}

PGDS was immunohistochemically demonstrated in all of the 10 arachnoid villi samples studied. The immunostaining pattern of arachnoid villi was different, however, from that of arachnoid: PGDS immunoreactivity was intense in core arachnoid cells rather than in the cap cell cluster and arachnoid barrier layer (Fig. 2A,C).

By immunoelectron microscopy, PGDS was also demonstrated to be localized at the rough ER membrane of core arachnoid cells (Fig. 2D).

Arachnoid cells in the villous core were ultrastructurally abundant with enlarged rough ER membrane (Fig. $2 F$ ).

\section{Meningiomas}

PGDS was immunohistochemically demonstrated in all of the 21 meningiomas studied; however, the extent of PGDS immunostaining was variable from case to case, and even in the same case from syncytium to syncytium, from whorl to whorl, and from psammoma to psammoma. Usually, PGDS immunoreactivity was intense in the tumor that included more syncytial portions, where 


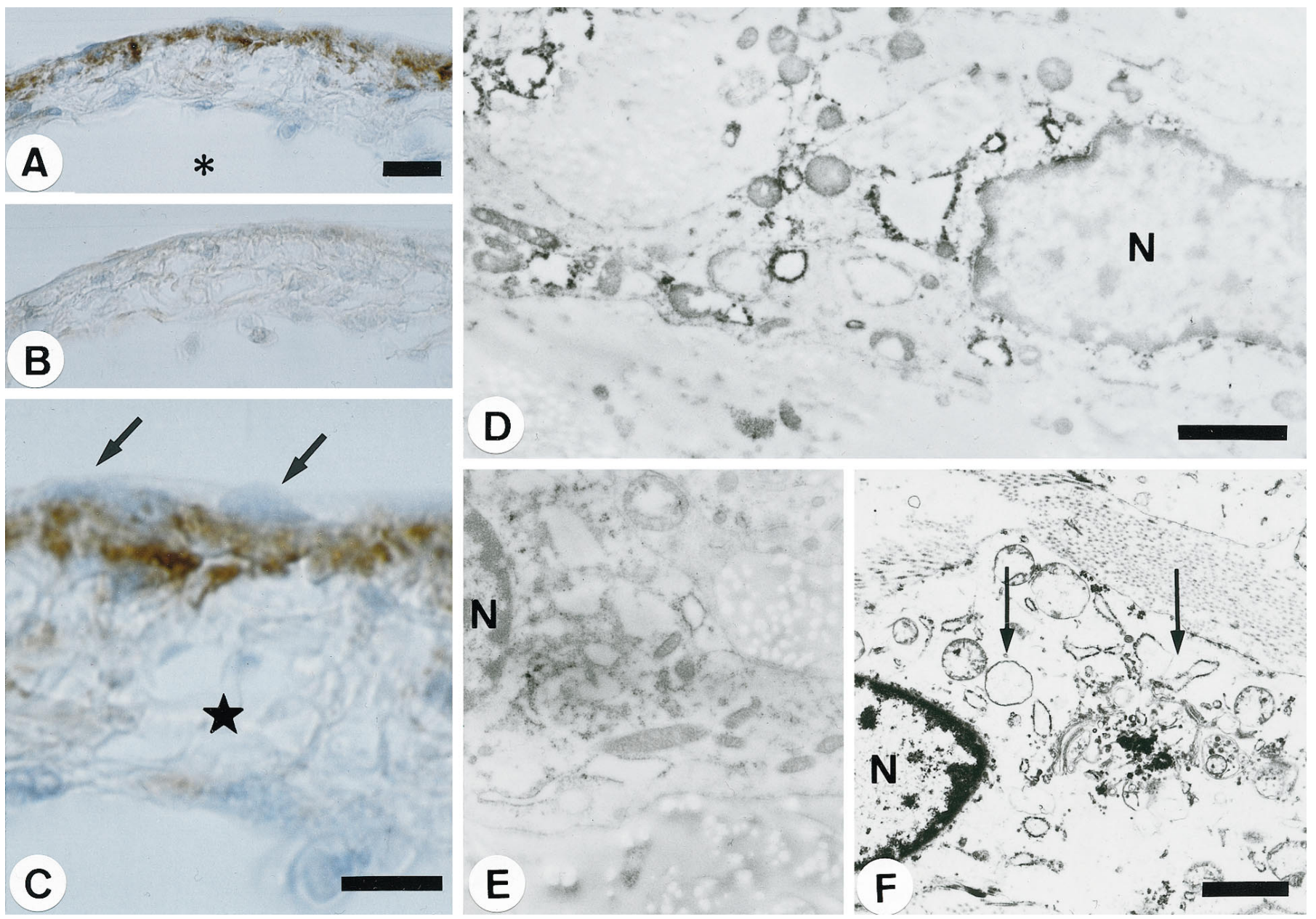

Figure 1. Immunohistochemical staining for PGDS in human arachnoid. $A$, Light microscopic low-power view shows intense granular DAB reaction products in arachnoid barrier cells overlying the subarachnoid space (asterisk). B, Control using preabsorbed antibody (same magnification as $A$ ). $C$, Higher magnification of $A$ reveals that both dural border cells (arrows) and the underlying arachnoid cells (star) show only weak immunoreactivity. $D$, Immunoelectron micrograph of arachnoid barrier cells shows PGDS at the rough ER membrane (no counterstaining), when it is compared with the conventional electron micrograph $F$. $N$, Nucleus. $E$, Control using nonimmunized rabbit serum (same magnification as $D$ ). $N$, Nucleus. $F$, Electron micrograph of an arachnoid barrier cell shows abundant rough ER membrane (arrows) in the electron-lucent cytoplasm (uranyl and lead staining). $N$, Nucleus. Scale bars: $A, 20 \mu \mathrm{m} ; C, 10 \mu \mathrm{m} ; D, 2 \mu \mathrm{m} ; F, 3 \mu \mathrm{m}$.

cell borders had disappeared and individual cells were no longer distinguishable. The immunoreactive deposits could be seen throughout the cytoplasm or were occasionally concentrated in the perinuclear region (Fig. 3C). Meningocytic whorls, where cells were arranged in a spiral-like structure, often showed an intense PGDS immunoreactivity (Fig. $3 A$ ), especially when they contained calcification deposits, the so-called psammoma bodies.

By immunoelectron microscopy, PGDS was also demonstrated to be localized at the rough ER membrane. PGDS often was concentrated in the perinuclear region as an aggregate of $\mathrm{DAB}$ reaction products (Fig. 3D).

Meningioma cells were ultrastructurally abundant with enlarged rough ER membrane (Fig. $3 F$ ), similar to arachnoid barrier cells (Fig. $1 F$ ) or core arachnoid cells (Fig. $2 F$ ).

Furthermore, in an additional immunohistochemical study, intense PGDS immunoreactivity was seen, especially in the outer aspects of spherical calcification corpuscles, psammoma bodies, or ossification deposits, which are sand-like centrically laminated, calcareous structures in the typical psammomatous (Fig. $4 A$ ) or ossifying meningiomas (Fig. 4B), although the surrounding tumor cells showed little staining.

\section{Control stainings}

The control immunohistochemical stainings of arachnoid (Fig. $1 B, E$ ), arachnoid villi (Fig. 2B,E), and meningiomas (Fig. 3B,E), using preabsorbed antibody and/or nonimmunized rabbit serum as primary antibodies, were negligible at both light and electron microscopic levels.

\section{Biochemical and molecular biological detection of PGDS}

As revealed by Western blot (Fig. 5), the extent of PGDS expression varied from case to case, although PGDS bands were specifically identified at $\sim 29 \mathrm{kDa}$ in all cases.

PCR was performed for the PGDS and GAPDH cDNA in parallel under the same conditions (Fig. 6). Both PGDS- and GAPDH-specific oligonucleotide primers generated a distinct single fragment of 342 and $600 \mathrm{bp}$, respectively, although it was weak in the fibroblastic type, presumably because of the small cell number. The PGDS gene showed an almost equal amount of expression in all cases, regardless of the histological subtypes and regardless of benign or malignant character. Arachnoid villi similarly showed PGDS gene expression. 


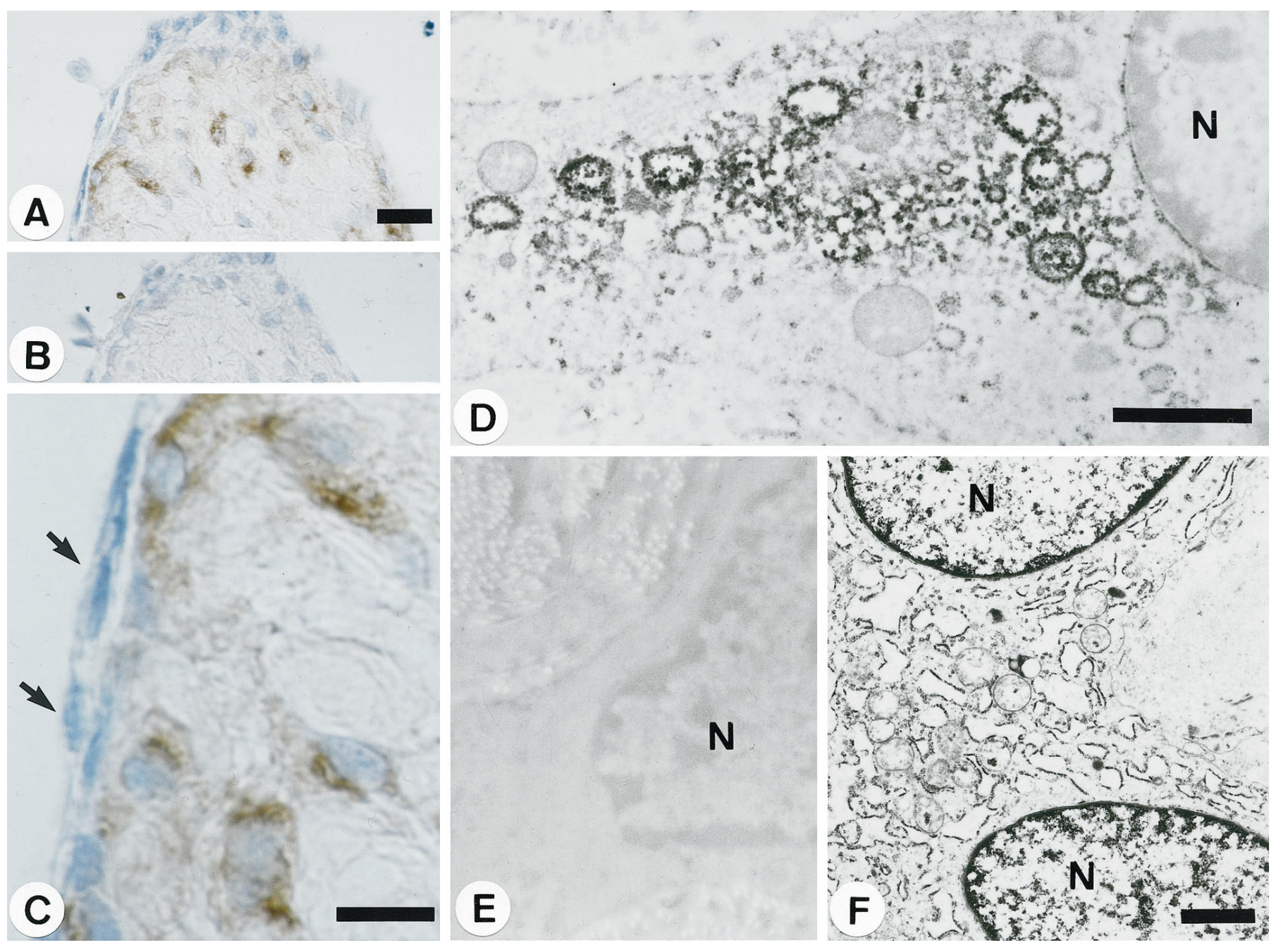

Figure 2. Immunohistochemical staining for PGDS in human arachnoid villi. $A$, Light microscopic low-power view shows intense DAB reaction products in the cytoplasm of core arachnoid cells. Note that the cap cell cluster shows very little immunoreaction. $B$, Control using preabsorbed antibody (same magnification as $A$ ). $C$, In contrast, higher magnification of $A$ reveals that the surrounding arachnoid cell layer (arrows) shows very little immunoreactivity ( $A-C$ counterstained with hematoxylin). $D$, Immunoelectron micrograph of a core arachnoid cell shows PGDS at the rough ER membrane (no counterstaining), when it is compared to the conventional electron micrograph $F$. N, Nucleus. $E$, Control using nonimmunized rabbit serum (same magnification as $D$ ). $N$, Nucleus. $F$, Electron micrograph of core arachnoid cells shows abundant rough ER membrane in the cytoplasm (uranyl and lead staining). $N$, Nuclei. Scale bars: $A, 20 \mu \mathrm{m} ; C, 10 \mu \mathrm{m} ; D, 2 \mu \mathrm{m} ; F, 2 \mu \mathrm{m}$.

\section{DISCUSSION}

\section{PGDS in arachnoid cells}

The present immunoelectron microscopic data, when compared with the conventional ultrastructural feature, indicate that PGDS is synthesized mainly at the rough ER membrane of arachnoid cells. This is consistent with the previous findings in oligodendrocytes of adult rats (Urade et al., 1987) and in the chick dorsal root ganglion cells (Vesin et al., 1995). In this study, PGDS immunoreactivity was seen mainly in arachnoid barrier cells rather than arachnoid trabecular cells in the subarachnoid space. The function of the arachnoid barrier layer separating the subarachnoid space from dura mater is obscure at present (Yamashima and Friede, 1984), and whether it contributes to CSF absorption or, on the contrary, to $\mathrm{CSF} /$ blood barrier is unknown. Interestingly, the immunolocalization of PGDS was distinct in arachnoid villi from arachnoid. It was seen mainly in the core arachnoid cells within the subarachnoid space rather than in the cap cell cluster or arachnoid cell layer. This difference in PGDS immunolocalization between arachnoid villi and arachnoid might be related to their distinct functions. The main function of arachnoid villi in humans is absorption of CSF. The high abundance of PGDS within core arachnoid cells might reflect a role of this protein in CSF absorption.

In rat leptomeninges, we found a different pattern of PGDS localization (N. Eguchi, C. T. Beuckmann, Y. Urade, and O. Hayaishi, unpublished results). In rat, PGDS is not located in arachnoid barrier cells but is located exclusively in arachnoid trabecular cells, which in humans do not possess PGDS. The intracellular localization of PGDS in rat arachnoid trabecular cells is the outer nuclear envelope and ER membrane. These findings may reflect an important aspect of functional difference in PGDS among species. Anatomically and histologically, human and rat leptomeninges differ remarkably. For instance, in rat arachnoid membrane only very few arachnoid villi can be found, and they do not show development of arachnoid cell layers or cap cell clusters. Furthermore, in contrast to human CSF, the bulk flow of CSF in rat is drained via a nasal route into the lymphatics 

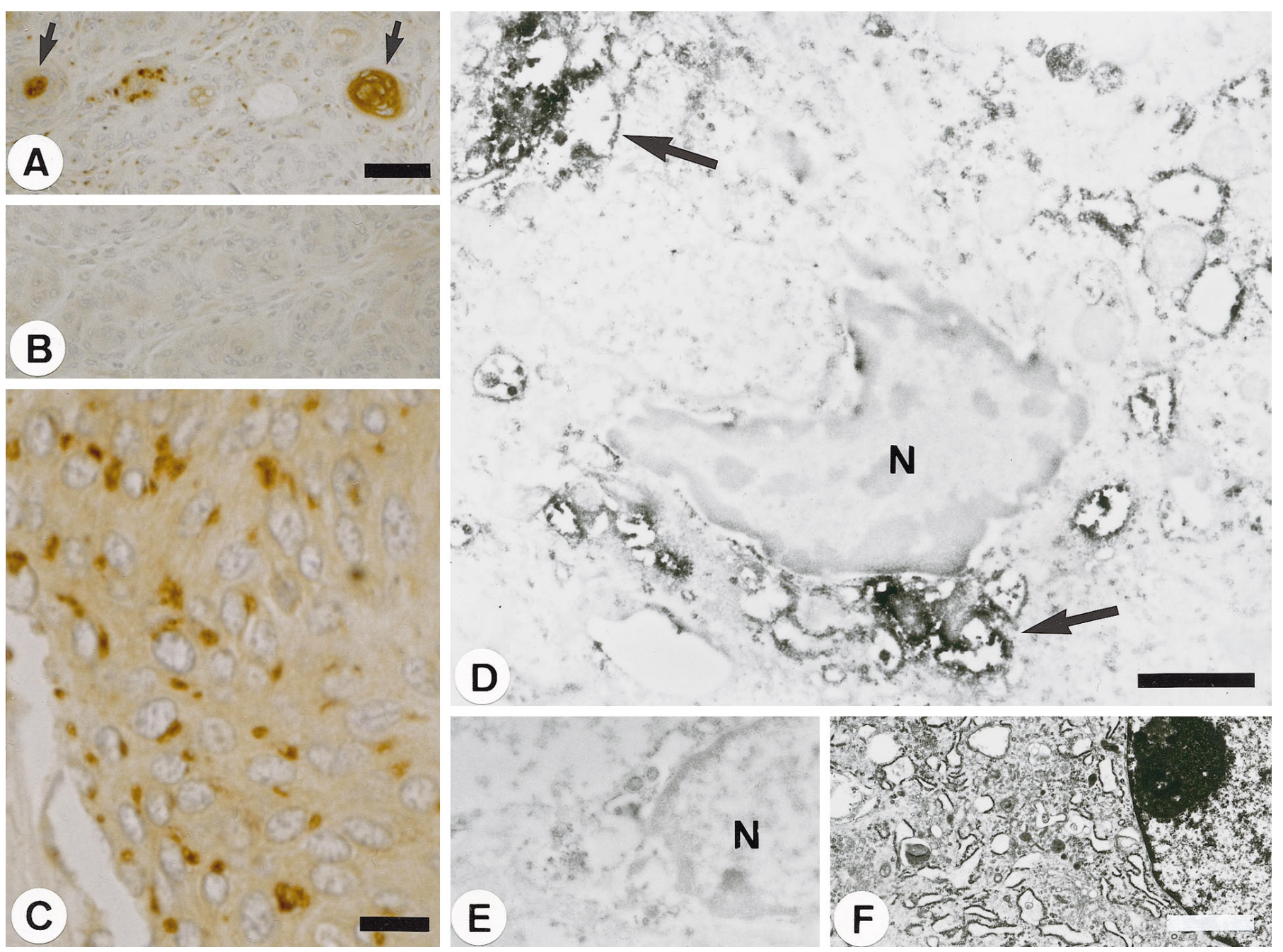

$\mathbf{N}$
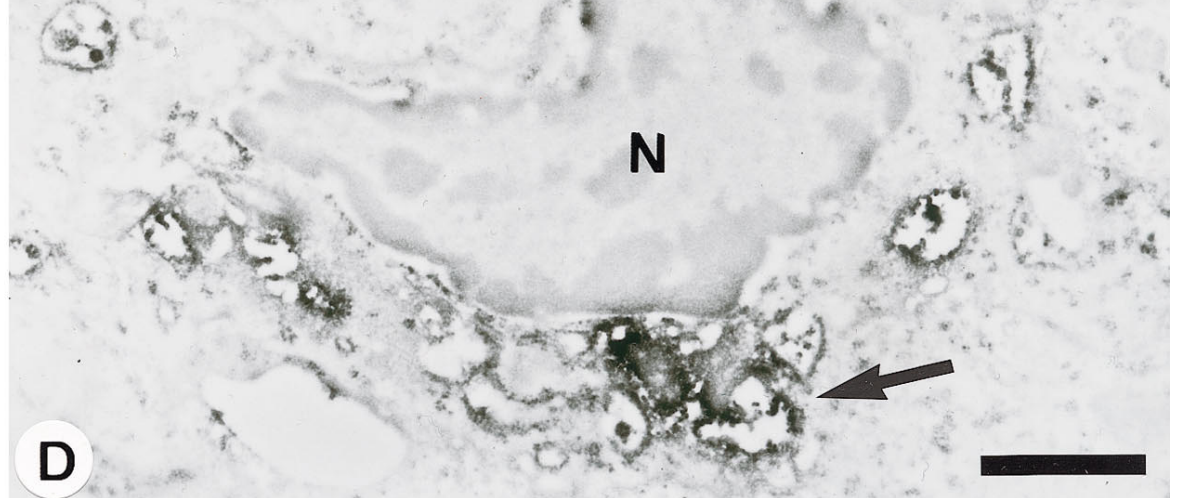

Figure 3. Immunohistochemical staining for PGDS in human meningioma of the syncytial type. $A$, At low magnification, intense DAB reaction products are seen within whorls (arrows). B, Control using preabsorbed antibody (same magnification as $A$ ). $C$, High-power light microscopic photograph reveals cytoplasmic PGDS immunoreactivity near cell nuclei $(A-C$ counterstained with hematoxylin). $D$, Immunoelectron micrograph of a meningioma cell shows PGDS at the rough ER membrane, when it is compared with the conventional electron micrograph $F$, and aggregates of DAB reaction products are seen within the cistern of the perinuclear region (arrows; no counterstaining). $N$, Nucleus. $E$, Control using nonimmunized rabbit serum (same magnification as $D$ ). $N$, Nucleus. $F$, Electron micrograph of a meningioma cell shows abundant rough ER membrane in the cytoplasm (uranyl and lead staining). $N$, Nuclei. Scale bars: $A, 200 \mu \mathrm{m} ; C, 50 \mu \mathrm{m} ; D, 2 \mu \mathrm{m} ; F, 3 \mu \mathrm{m}$.

(Kida et al., 1993). Therefore, care should be taken in comparing different species in regard to localization and function of PGDS.

\section{PGDS in meningioma cells}

The roles of PGDS in human meningiomas are unclear at present, but their further characterization might allow a clear understanding of these tumors. The present study demonstrated abundant expression and biosynthesis of PGDS in human meningioma cells. Although the extent of PGDS expression and immunoreactivity varied from case to case, an almost equal amount of amplified PGDS gene was found in all of the histological subtypes of meningiomas, regardless of benign or malignant character. Because human brain tumors such as malignant astrocytomas (five cases), oligodendrogliomas (three), acoustic neurinomas (three), ependymomas (three), pituitary adenomas (three), craniopharyngiomas (three), hemangioblastomas (three), metastatic brain tumors (three), and choroid plexus papilloma (one) showed negligible PGDS immunoreactivity in our additional study (data not shown) using the same antibody, PGDS can be considered a specific cell marker of meningiomas.
Yamashima and Yamashita (1990) previously found abundant extra-membraneous multilamellar bodies among and within meningioma cells by electron microscopy using tannic acid mixed with conventional fixatives. It is tempting to speculate that these multilamellar bodies might have some relation to membrane-derived arachidonic acid, from which $\mathrm{PGD}_{2}$ is synthesized with the aid of PGDS through arachidonate cascades. Not only PGDS but also glutathione$S$-transferase were reported to catalyze the conversion of $\mathrm{PGH}_{2}$ to $\mathrm{PGD}_{2}$ (Ujihara et al., 1988). Hara et al. (1990) identified immunohistochemically glutathione- $S$-transferase of the placental type in human meningiomas. Accordingly, in meningiomas, both PGDS and glutathione- $S$-transferase are capable of catalyzing the reaction to produce $\mathrm{PGD}_{2}$. This prostanoid was reported to regulate growth and differentiation of rat glioma C6BU-1 cells and to induce $\mathrm{Ca}^{2+}$ influx and cGMP formation in mouse neuroblastoma-rat glioma hybrid NG108-15 cells (Hayaishi, 1988; Ito et al., 1989). The interaction of $\mathrm{PGD}_{2}$ with growth and differentiation of meningiomas should be studied further to elucidate the possible role of $\mathrm{PGD}_{2}$ in meningioma tumorigenesis. 


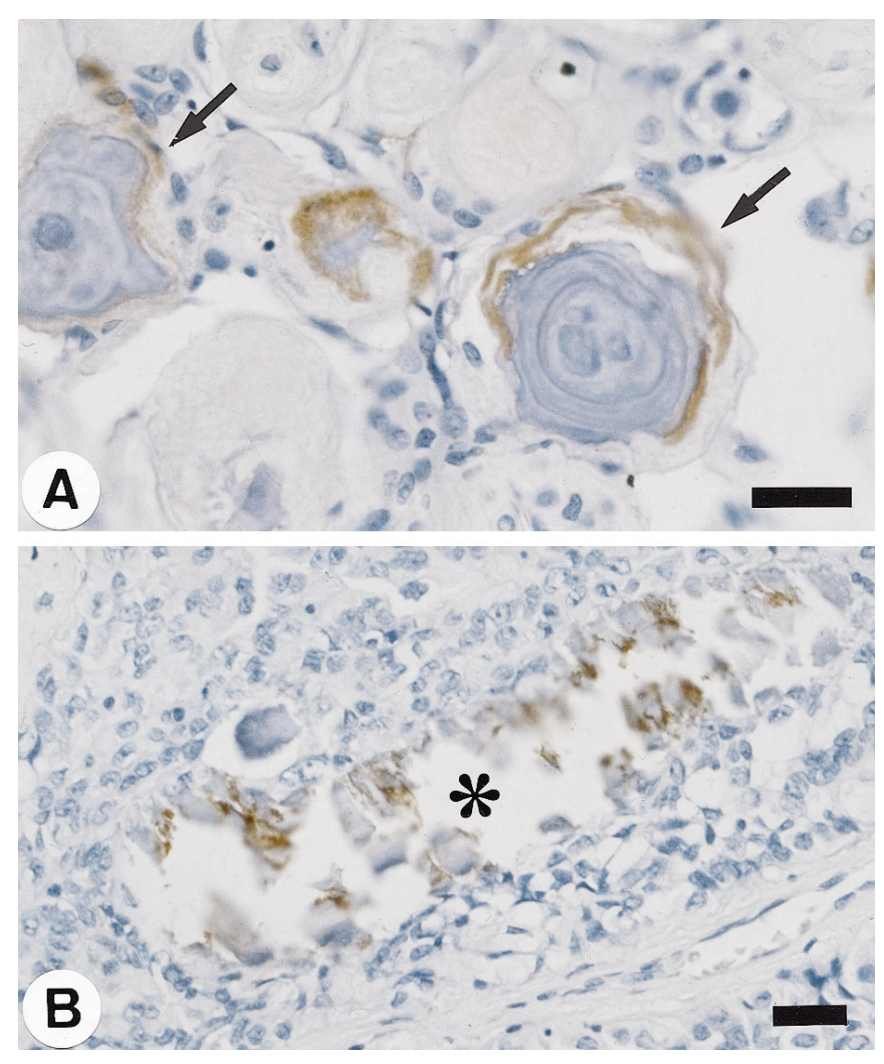

Figure 4. Immunohistochemical staining for PGDS in human psammomatous meningioma or ossifying meningioma. $A$, In psammomatous meningioma, intense $\mathrm{DAB}$ reaction products are detected, especially in the outer aspects of psammoma bodies (arrows). B, Another example of PGDS localization in ossification deposits (asterisk) in ossifying meningioma. ( $A$ and $B$ counterstained with hematoxylin.) Scale bars, $100 \mu \mathrm{m}$.

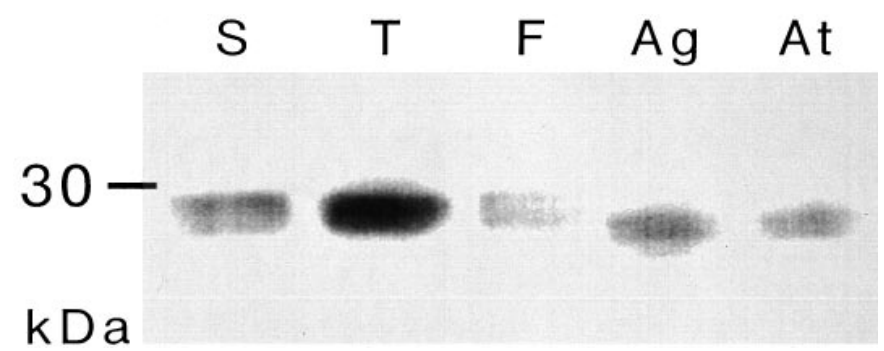

Figure 5. Western blot analysis for PGDS in human meningiomas. PGDS is expressed to a variable extent in various types of meningiomas $(S$, syncytial; $T$, transitional; $F$, fibroblastic; $A g$, angiomatous; $A t$, atypical). The position of molecular weight marker is indicated at the left.

Interestingly, the PGDS immunolocalization in meningiomas was often concentrated both within meningocytic whorls containing calcification deposits and at the hot calcification or ossification sites around psammoma bodies (Fig. 4). It was already demonstrated that there are numerous precursors of psammoma bodies in the vicinity of immature psammoma bodies and within meningocytic whorls in arachnoid villi (Yamashima et al., 1986) and meningiomas (Kubota et al., 1986). Taken together that in arachnoid villi or meningiomas intense PGDS immunoreactivities were concentrated at the calcifying or ossifying sites in psammoma bodies or whorls, it is suggested that PGDS and the resultant $\mathrm{PGD}_{2}$ may have some relation to calcification/ossification processes. Furthermore, if one considers that meningiomas are often
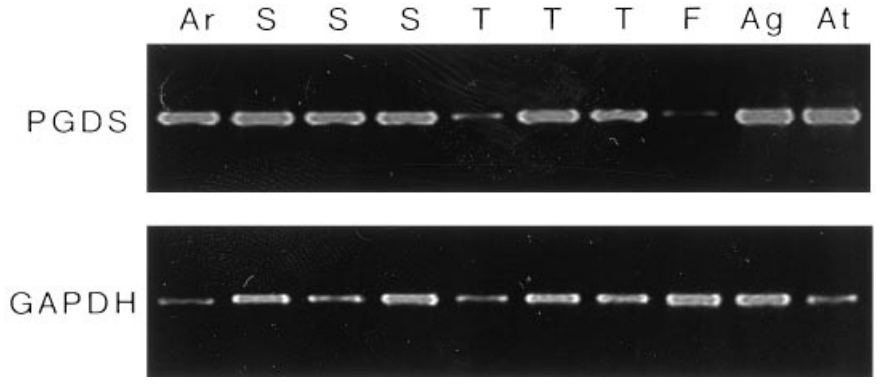

Figure 6. RT-PCR analysis for PGDS gene in human arachnoid villi and meningiomas. PGDS gene is amplified by RT-PCR, and its expression of 342 bp (top) is observed consistently in arachnoid villi $(A r)$ and all meningioma cases ( $S$, syncytial; $T$, transitional; $F$, fibroblastic; $A g$, angiomatous; $A t$, atypical types). As an internal control, the expression of GAPDH gene of $600 \mathrm{bp}$ (bottom) is also shown.

associated with hyperostosis of the adjacent bone, it is tempting to speculate that PGDS being secreted from the arachnoid or the tumor is related to the formation and metabolism of the skull.

\section{Dual function of PGDS}

Finally, the present data point out that human arachnoid and meningioma cells can serve as an excellent material to study both the function and metabolism of $\mathrm{PGD}_{2}$ and the bifunctional ability of PGDS as a $\mathrm{PGD}_{2}$-producing enzyme and a potential lipophilic ligand transporter, as proposed previously (Urade, 1996). PGDS belongs to the gene superfamily of lipocalins (Nagata et al., 1991). Lipocalins are transport proteins carrying small lipophilic molecules (Pervaiz and Brew, 1987). PGDS binds retinoids with affinities comparable to other retinoid-transporter proteins in vitro. It was therefore suggested to have a dual function, being an enzyme as well as a transporter protein (Urade, 1996).

To clarify the function of PGDS in the human brain and meningiomas, possible lipophilic candidates as a ligand for this transport enzyme should be determined. The outcome of possible candidates should be studied further with particular attention paid to the function of arachnoid cells or arachnoid villi, differentiation of meningioma cells, and participation in calcification or ossification processes. The possibility should also be considered that $\mathrm{PGD}_{2}$ itself is attached to the enzyme of its synthesis, transferred with the circulating CSF, and then taken up by neurons or glial cells where it might elicit a function.

\section{REFERENCES}

Beuckmann CT, Gordon WC, Kanaoka Y, Eguchi N, Marcheselli VL, Gerashchenko DY, Urade Y, Hayaishi O, Bazan NG (1996) Lipocalintype prostaglandin D synthase ( $\beta$-trace) is located in pigment epithelial cells of rat retina and accumulates within interphotoreceptor matrix. J Neurosci 16:6119-6124.

Chomczynski P, Sacchi N (1987) Single-step method of RNA isolation by acid guanidinium thiocyanate-phenol-chloroform extraction. Anal Biochem 162:156-159.

Clausen J (1961) Proteins in normal cerebrospinal fluid not found in serum. Proc Soc Exp Biol Med 107:170-172.

Hara A, Yamada H, Sakai N, Hirayama H, Tanaka T, Mori H (1990) Immunohistochemical expression of glutathione $S$-transferase placental type (GST- $\pi$ ), a detoxifying enzyme, in normal arachnoid villi and meningiomas. Virchows Arch [A] 6:493-496.

Hayaishi O (1988) Sleep-wake regulation by prostaglandins $D_{2}$ and $E_{2}$. J Biol Chem 263:14593-14596.

Hayaishi O (1994) Tryptophan, oxygen, and sleep. Annu Rev Biochem 63:1-24.

Hoffmann A, Conradt HS, Gross G, Nimtz M, Lottspeich F, Wurster U (1993) Purification and chemical characterization of $\beta$-trace protein 
from human cerebrospinal fluid: its identification as prostaglandin D synthase. J Neurochem 61:451-456.

Ito S, Narumiya S, Hayaishi O (1989) Prostaglandin $\mathrm{D}_{2}$ : a biochemical perspective. Prostaglandins Leukot Essent Fatty Acids 37:219-234.

Kida S, Pantazis A, Weller RO (1993) CSF drains directly from the subarachnoid space into nasal lymphatics in the rat: anatomy, histology and immunological significance. Neuropathol Appl Neurobiol 19:480-488.

Kubota T, Yamashima T, Hasegawa M, Kida S, Hayashi M, Yamamoto S (1986) Formation of psammoma bodies in meningocytic whorls: ultrastructural study and analysis of calcified material. Acta Neuropathol 70:262-268.

Kuruvilla AP, Hochwald GM, Ghiso J, Castano EM, Pizzolato M, Frangione B (1991) Isolation and amino terminal sequence of $\beta$-trace, a novel protein from human cerebrospinal fluid. Brain Res 565:337-340.

Matsumura H, Nakajima T, Osaka T, Satoh S, Kawase K, Kubo E, Kantha SS, Kasahara K, Hayaishi O (1994) Prostaglandin $D_{2}$-sensitive, sleeppromoting zone defined in the ventral surface of the rostral basal forebrain. Proc Natl Acad Sci USA 91:11998-12002.

Nagata A, Suzuki Y, Igarashi M, Eguchi N, Toh H, Urade Y, Hayaishi O (1991) Human brain prostaglandin D synthase has been evolutionarily differentiated from lipophilic-ligand carrier proteins. Proc Natl Acad Sci USA 88:4020-4024.

Onoe H, Ueno R, Fujita I, Nishino H, Oomura Y, Hayaishi O (1988) Prostaglandin $\mathrm{D}_{2}$, a cerebral sleep-inducing substance in monkeys. Proc Natl Acad Sci USA 85:4082-4086.

Peitsch MC, Boguski MS (1991) The first lipocalin with enzymatic activity. Trends Biochem Sci 16:363.

Pervaiz S, Brew K (1987) Homology and structure-function correlations between $\alpha_{1}$-acid glycoprotein and serum retinol-binding protein and its relatives. FASEB J 1:209-214.

Ueno R, Honda K, Inoue S, Hayaishi O (1983) Prostaglandin $\mathrm{D}_{2}$, a cerebral sleep-inducing substance in rats. Proc Natl Acad Sci USA 80:1735-1737.
Ujihara M, Urade Y, Eguchi N, Hayashi H, Ikai K, Hayaishi O (1988) Prostaglandin $\mathrm{D}_{2}$ formation and characterization of its synthetases in various tissues of adult rats. Arch Biochem Biophys 260:521-531.

Urade Y (1996) Dual function of $\beta$-trace. Prostaglandins 51:286.

Urade Y, Fujimoto N, Hayaishi O (1985) Purification and characterization of rat brain prostaglandin $\mathrm{D}$ synthase. $\mathrm{J}$ Biol Chem 260:12410-12415.

Urade Y, Fujimoto N, Kaneko T, Konishi A, Mizuno N, Hayaishi O (1987) Postnatal changes in the localization of prostaglandin D synthase from neurons to oligodendrocytes in the rat brain. J Biol Chem 262:15132-15136.

Urade Y, Kitahama K, Ohishi H, Kaneko T, Mizuno N, Hayaishi O (1993) Dominant expression of mRNA for prostaglandin D synthase in leptomeninges, choroid plexus, and oligodendrocytes of the adult rat brain. Proc Natl Acad Sci USA 90:9070-9074.

Vesin MF, Urade Y, Hayaishi O, Droz B (1995) Neuronal and glial prostaglandin D synthase isozymes in chick dorsal root ganglia: a light and electron microscopic immunocytochemical study. J Neurosci $15: 470-476$

Watanabe K, Urade Y, Mäder M, Murphy C, Hayaishi O (1994) Identification of $\beta$-trace as prostaglandin D synthase. Biochem Biophys Res Commun 203:1110-1116.

Yamashima T, Friede RL (1984) Light and electron microscopic studies on the subdural space, the subarachnoid space and the arachnoid membrane. Neurol Med Chir (Tokyo) 24:737-746.

Yamashima T, Yamashita J (1990) Histological, ultrastructural, and chromatographical discrimination of phospholipids in meningiomas. Acta Neuropathol 80:255-259.

Yamashima T, Kida S, Kubota T, Yamamoto S (1986) The origin of psammoma bodies in the human arachnoid villi. Acta Neuropathol 71:19-25.

Zahn M, Mäder M, Schmidt B, Bollensen E, Felgenhauer K (1993) Purification and $\mathrm{N}$-terminal sequence of $\beta$-trace, a protein abundant in human cerebrospinal fluid. Neurosci Lett 154:93-95. 